\title{
Gestión de aula ante Conductas Contrarias a la Convivencia en Educación Secundaria Obligatoria Classroom management in problematic behaviors of coexistence in mandatory secondary education Francisco Javier Gil Espinosa, Palma Chillón Garzón, Miguel Ángel Delgado Noguera Universidad de Granada (España)
}

Resumen. Los problemas de convivencia y disciplina en la Educación Secundaria Obligatoria son un discurso generalizado y, consecuentemente, se requiere diagnosticar esta situación y desarrollar pautas de actuación que mejoren la convivencia en el aula. El objetivo del presente trabajo es identificar las principales medidas que el profesorado dispone ante problemáticas conductuales del alumnado en Educación Secundaria Obligatoria con el objetivo de propiciar una mejora del clima de convivencia y gestión del aula. Los participantes son 147 profesores de Educación Física que ejercen activamente la profesión en centros de Educación Secundaria Obligatoria. Dicha muestra cumplimentó dos cuestionarios diseñados «ad hoc» para evaluar las conductas contrarias a la convivencia en el alumnado. En el análisis estadístico se aplicó chi-cuadrado para relacionar las conductas con otras variables de interés. Los resultados de este estudio nos orientan hacia la utilización de diferentes medidas de gestión del aula en función de la conducta contraria a la convivencia que surja. Por otro lado, variables como la antigüedad en el cuerpo y el sexo del profesor han resultado determinantes en el tipo de medidas aplicadas. Este trabajo aporta una propuesta de actuación genérica ante las conductas contrarias a la convivencia con el objetivo de intervenir de forma progresiva, integradora y eficaz.

Palabras clave. Gestión de aula, Conflicto, Convivencia, Educación Secundaria Obligatoria.

\begin{abstract}
The problems of discipline and coexistence in class are a general concern in mandatory secondary education. Consequently, it is essential to identify the causes and establish action guidelines that can improve the coexistence of students in class. The purpose of this research is to identify the main actions that could be applied by teachers when students in mandatory secondary education engage in problematic behaviors in order to improve the coexistence atmosphere and classroom management. The participants are 147 instructors of Physical Education who are teaching actively in institutions of mandatory secondary education. Participants completed two questionnaires created 'ad hoc' to evaluate the students' problematic behaviors regarding coexistence in these classes. The statistical analysis we used was the chi-squared test to associate these behaviors to other variables of interest. The results of this research provide guidance for the use of different classroom management techniques considering such problem behaviors. Furthermore, variables such as the years of work experience and the teacher gender were critical regarding the type of actions implemented. This work provides a proposal for intervention in the case of problem behaviors with the aim to be progressive, inclusive and effective.

Key Words. Classroom management, Conflict, School Coexistence, Mandatory Secondary Education.
\end{abstract}

\section{Introducción}

El análisis pedagógico en los últimos años ha comenzado a reconsiderar la propuesta de que los docentes y la forma en que realizan su trabajo son piezas clave de cualquier estrategia eficaz dentro de una reforma educativa (Penalva, Hernández \& Guerrero, 2013; Szymanska \& Timmermans, 2007). Del Rincón (2002) considera al educador la variable más importante que configura el contexto escolar. El profesor debe preparar un entorno suficientemente enriquecedor con el que el alumno pueda interaccionar y convertirse en el artífice de su propio crecimiento, como individuo y como ser social.Así, las arraigadas normas internacionales muestran la importancia de que los educadores dispongan de un buen material y situación social como variable clave para una Educación de calidad (Comité Mixto OIT/UNESCO-CEART-, 1996).

Por otrolado, sonnumerosos los estudios que señalan el descontento del profesoradoy la comunidad educativa respecto al clima de convivencia que existe en la mayoría de los centros de Educación Secundaria Obligatoria (ESO) a nivel nacional (Aznar, Cáceres \& Hinojo, 2007; Buscà, Ruiz \& Rekalde, 2014; Capllonch, Figueras \& Lleixà 2014; Cerezo, 2007; Estévez, 2005; Fernández, 2015; Figueras, Calvo \& Capllonch, 2014; Luengo \& Sánchez, 2006; Moreno, Cervelló, Martínez \& Alonso, 2007; Muñoz, Carreras \& Braza, 2004), así como en otros países (McCormack, 1997; Blaya, Debarbieux, Rey \& Ortega, 2006; Gavotto, 2015; Mejía \& Urrea, 2015).

Moreno et al. (2007, p.170), citando a Sáenz-López (1997), indican que «la dirección de la clase abarca numerosos aspectos, siendo uno de los más relevantes el que hace referencia a la organización y la disciplina, ya que sin esta no se podrán llevar a cabo los objetivos planteados...». En la misma línea, Delceva (2014) y Macías \& Ariel (2015) concluyen que existe un déficit de habilidades de gestión de aula motivado por carencias en la formación inicial del profesorado. Villalta (2014) indica que la relación entre bajo nivel socioeconómico y escaso rendimiento escolar ha sido rota en ocasiones debido a factores asociados al aprendizaje

Fecha recepción: 06-12-15. Fecha de aceptación: 20-01-16 Francisco Javier Gil Espinosa

fjgesierraluna@gmail.com tales como aspectos organizativos, de gestión directiva o de calidad de la docencia que, entre otros aspectos, son constitutivos de dinámicas institucionales de las llamadas Escuelas eficaces. Por tanto, la formación y competencias del docente podrían condicionar el modelo de escuela, el proceso de enseñanza aprendizaje y, consecuentemente, la prevención, tratamiento y resolución de conflictos o Conductas Contrarias a la Convivencia (CCC) (Bancotovska, 2015; Cassullo \& García, 2015; Vallory, 2015).

Considerando que «gestionar» es definido por la Real Academia Española(RAE)(2015) como llevar adelante una iniciativa o un proyecto así como manejar o conducir una situación problemática, se entiende en el estudio como "gestión de aula» el proceso mediante el cual el profesorado, en el aula, lleva adelantela enseñanza-aprendizajemanejando adecuadamentelas situaciones problemáticas. Simultáneamente debemos considerar el concepto de «Estrategia Didáctica» pues la RAE (2015) define estrategia como el arte de dirigir las operaciones militares, y en una segunda acepción como arte, traza para dirigir un asunto. Adaptada a la enseñanza, es el «arte de dirigir las operaciones de la enseñanza» (Delgado, 1991, p.10). A su vez, el término de estrategia didáctica es definido como «el conjunto de las acciones que realiza el docente con clara y explícita intencionalidad pedagógica» (Rosales, 2004, p.1). Esta concepción atribuye al docenteuna importancia decisiva como orientador, guía y facilitador del aprendizajemediante un adecuado accionar. Rajadell (2002, p.1) utiliza el término de estrategia de intervención didáctica, y lo define como una «actuación secuenciada potencialmente consciente del profesional en Educación, guiada por uno o más principios de la didáctica, y encaminada hacia la optimización del proceso de enseñanzaaprendizaje».

A pesar de la importancia de la gestión del aula en el contexto de crear situaciones de aprendizaje exitosas, existe falta de información sobre las medidas que el profesorado adopta y si estas son realmente efectivas en el control de la disciplina. Por tanto, el presente estudio tiene como objetivos: (a) conocer la frecuencia de utilización y efectividad percibida de las medidas adoptadas por el profesorado ante CCC en clase; (b) conocer la frecuencia de utilización y efectividad percibida de las medidas adoptadas por el profesorado para cada uno de los factores de CCC, y (c) examinar las medidas adoptadas en función de las características del profesor. 


\section{Método}

\section{Diseño de la investigación}

El diseño de la investigación se basó en un estudio transversal de base poblacional mediante la aplicación de cuestionarios dirigidos al profesorado de Educación Física (EF) que imparta niveles de ESO en centros públicos de la Comunidad Autónoma deAndalucía.

\section{Criterios de inclusión}

Los criterios de inclusión de los participantes fueron dos:

1. Ser profesor de EF en activo durante el curso académico 2007/ 2008 en un centro público que imparta la etapa de ESO del territorio de Andalucía.

2. Haber cumplimentado, al menos, los datos básicos del primer cuestionario (para que fuese posibleel análisis de las variables del estudio) y completamente el segundo cuestionario (que versa sobre las medidas aplicadas ante la aparición de CCC en clase).

\section{Muestra}

La población objeto de estudio son los profesores y profesoras del área de EF que trabajan en centros públicos andaluces. De un total de 844 centros se recibieron 147 cuestionarios de 110 centros distintos, de los cuales 82 cuestionarios son de 59 centros situados en localidades de tres o más centros, 46 cuestionarios de 38 centros de las capitales de provincia y 19 cuestionarios de 13 centros de localidades con uno o dos centros.

El tamaño de la muestra fue de 147 sujetos, que para el supuesto muestra aleatoria simple (MAS), y de máxima indeterminación $p=q=50 \%$, y para un nivel de confianza del 95\% supone un error muestral máximo de +/-8\%. Debemos señalar el tamaño muestral como una limitación del estudio que no permite generalizar los resultados pero que no altera los resultados del estudio, dada la naturaleza de sus objetivos.

Teniendo en cuenta los criterios anteriores, en la tabla 1 se muestra el número de centros a los que se enviaron los cuestionarios por correo postal, distribuidos por provincias. Suman un total de 471 envíos, sobre 844 centros.

$\begin{aligned} & \text { Tabla } 1 \\
& \text { Número de centros a los que se enviaron los cuestionarios por correo postal, distribuidos } \\
& \text { por provincias. }\end{aligned}$
\begin{tabular}{lcc}
\hline Provincia & $\mathbf{N}^{\circ}$ centros totales & $\mathbf{N}^{\circ}$ centros enviados \\
\hline Almería & 75 & 39 \\
Cádiz & 125 & 105 \\
Córdoba & 88 & 41 \\
Granada & 93 & 40 \\
Huelva & 59 & 16 \\
Jaén & 87 & 26 \\
Málaga & 131 & 96 \\
Sevilla & 186 & 108 \\
\hline Total Andalucía & $\mathbf{8 4 4}$ & $\mathbf{4 7 1}$
\end{tabular}

\section{Instrumento de recogida de datos: cuestionarios}

Los instrumentos utilizados han sido dos cuestionarios diseñados específicamente para tal fin. El cuestionario 1 denominado «Estrategias utilizadas por el centro y el profesor de Educación Física en relación con la Convivencia (EUCEFC)» nos ofrece datos de los participantes para el presente estudio. El cuestionario 2 denominado «Medidas adoptadas ante Conductas Contrarias a la Convivencia y Participación en clase de Educación Física (MA3CPEF)» recoge las medidas más frecuentes y se contempla la opción de introducir alguna nueva por los encuestados.

La elaboración de estos cuestionarios comenzó con una revisión bibliográfica en busca de los factores condicionantes de la convivencia en los centros educativos así como de investigaciones similares, tras la cual se elaboró una propuesta inicial para ser revisada por siete expertos, personas representativas por su formación y profesión en relación a la convivencia escolar, dos profesores universitarios de Ciencias de la Educación, un profesor universitario de Ciencias de la Actividad Física y el Deporte, una Doctora e inspectora de Educación, una Doctora y Orientadora deEducación Secundaria, un Director deCentro de formación de profesorado y un profesor de Educación Secundaria de Educación Física. Los cuestionaros fueron rediseñados con las mejoras propuestas por el grupo de expertos antes de proceder a la última fase, de aplicación a compañeros profesores y profesoras de ESO, como estudio piloto.
Finalmente, se analizaron los resultados del estudio piloto al objeto de finalizar la redacción de los instrumentos de recogida de datos, y se obtuvieron los cuestionarios definitivos.

\section{Procedimiento de recogida de datos}

Se enviaron los cuestionarios a cada uno de los centros públicos de localidades andaluzas con tres o más centros de ESO por correo postal, a la atención del Departamento de EF, incluyendo un sobre franqueado y con datos de vuelta cumplimentados para su devolución, junto a la carta de presentación.

Al resto de centros, de localidades con uno o dos centros de ESO, se les envió por correo electrónico, dando la opción de contestar en archivo electrónico, en una aplicación diseñada «en línea» o bien imprimiendo los cuestionarios o solicitándolos, para su envío por correo postal.

A la semana de haber sido enviados los cuestionarios se envió un correo electrónico a los centros, a la atención del Departamento de EF, presentando e informando del envío de los mismos. Con posterioridad al envío del correo electrónico se contactó telefónicamente con alguno de los miembros del Departamento de EF de los centros que no habían confirmado recepción o enviado respuesta.

\section{Análisis Estadístico}

Se realizó una descripción de las características del profesorado y de su centro, para lo cual en las variables cualitativas se describieron las frecuencias absolutas y relativas y para las cuantitativas se calcularon los valores medios y la desviación típica (DT)

Las medidas aplicadas fueron descritas mediante análisis de frecuencias realizando un análisis factorial para reducción de los datos y realización de un análisis de la dimensionalidad. De esta manera se pudieron identificar un número menor de factores que explican la mayoría de la varianza observada. Se calculó el estadístico KMO para comprobar la adecuación de los datos al modelo factorial, y el contraste de Barlett para comprobar que las variables no están incorrelacionadas, asumiendo así que tiene sentido aplicar el análisis factorial. Como método de rotación se usó el método de rotación ortogonal VARIMAX. Igual se realizó con las CCC.

A cada medida, se le calcularon además los valores de efectividad media percibida. El rango de efectividad oscila entre un mínimo de 1 y un máximo de 4 en base a este criterio: 1, poco efectiva; 2 , algo efectiva; 3 , bastante efectiva y 4, muy efectiva.

Para describir las medidas adoptadas en función de las CCC se usaron los datos agregados por los factores de resultados de los análisis factoriales.

Además, se estudiaron las CCC y medidas aplicadas atendiendo a algunas variables de interés como el género del profesor y la antigüedad en el cuerpo. En dicho análisis bivariante se aplicó el test de chi-cuadrado o el test exacto de Fisher cuando las frecuencias esperadas de las casillas de la tabla de contingencia eran demasiado bajas. Se tomaron como valores «p» significativos los valores menores que .05 .

Los datos se grabaron y procesaron a través del programa estadístico «Statistical Package for Social Science» (SPSS) versión 15.0.

\section{Resultados}

Frecuencia y efectividad percibida de las medidas adoptadas por el profesorado

Es importante destacar que un 98\% del profesorado participante en esta investigación considera importante o muy importante lautilización de estrategias didácticas adecuadas para llevar a cabo la clase de EF con menos problemáticas de Convivencia. Es por ello que la formación y actualización docente debe ser continua y eficaz, en base a que los conflictos son parte integrante de la profesión y el profesor debe disponer y conocer estrategias y líneas de actuación sobre los factores que intervienen en el proceso de enseñanza-aprendizaje.

Las medidas adoptadas con mayor frecuencia han sidola de «penaliza la nota» (76.61\%), «llama al alumno por su nombre o le pregunta» 
(75.81\%), «establece charlas individuales» (74.19\%) y «se calla hasta que los alumnos bajen el tono» (74.19\%).

Las medidas que muestran una mayor efectividad han sido «castiga sin recreo o final de la jornada» (3.35), «llama personalmente al padre/ tutor» (3.25), «nombrar responsables para cada sesión» (3.19) y «castiga con trabajo físico» (3.15). La tabla 2 muestra la frecuencia con la que se toman las distintas medidas ante los problemas de conducta y la efectividad media de cada una de las medidas.

Tabla 2

Frecuencia y efectividad media percibida de las medidas adoptadas ante los problemas de conductas.

\begin{tabular}{lccc}
\hline \multicolumn{1}{c}{ Medidas adoptadas } & \multicolumn{3}{c}{ Frecuencia } \\
& n & $\begin{array}{c}\text { Efectividad } \\
\text { media } \\
\text { (1-4) }\end{array}$ \\
\hline 1 Penaliza la nota (poner un negativo, poner un "0", bajar la nota) & 95 & 76.61 & 2.78 \\
2 Llama al alumno por su nombre o le pregunta & 94 & 75.81 & 2.85 \\
3 Establece charlas individuales & 92 & 74.19 & 2.83 \\
4 Se calla hasta que los alumnos bajen el tono & 92 & 74.19 & 3.13 \\
5 Redacta un parte & 83 & 66.94 & 2.86 \\
6 Lo explica al tutor para su tratamiento & 81 & 65.32 & 2.55 \\
7 Reflexiona sobre la conducta y las normas una vez establecido el orden & 77 & 62.10 & 2.68 \\
8 Envía a la Jefatura de Estudios & 74 & 59.68 & 3.12 \\
9 Mira fijamente o hace algún gesto al alumno & 74 & 59.68 & 2.88 \\
10 Expulsión temporal de clase & 65 & 52.42 & 3.07 \\
11 Castiga con trabajo escrito & 61 & 49.19 & 2.79 \\
12 Diseña actividades que faciliten la integración del alumno & 60 & 48.39 & 2.88 \\
13 Actúa como mediador & 59 & 47.58 & 2.74 \\
14 Refuerza positivamente al alumno que actúa correctamente & 59 & 47.58 & 3.05 \\
15 Grita por encima de los alumnos hasta restablecer el orden & 53 & 42.74 & 2.83 \\
16 Llama personalmente al padre/tutor & $\mathbf{4 8}$ & $\mathbf{3 8 . 7 1}$ & $\mathbf{3 . 2 5}$ \\
17 Cambia a los alumnos de sitio & 41 & 33.06 & 2.85 \\
18 Deriva al departamento de orientación & 34 & 27.42 & 2.60 \\
19 Nombra responsables para cada sesión & $\mathbf{3 4}$ & $\mathbf{2 7 . 4 2}$ & $\mathbf{3 . 1 9}$ \\
20 Castiga sin recreo o final de la jornada & $\mathbf{3 2}$ & $\mathbf{2 5 . 8 1}$ & $\mathbf{3 . 3 5}$ \\
21 Realiza contrato con el alumno y/o familia & 26 & 20.97 & 2.79 \\
22 Ignora el hecho & 23 & 18.55 & 1.99 \\
23 Castiga con trabajo físico & $\mathbf{1 5}$ & $\mathbf{1 2 . 1 0}$ & $\mathbf{3 . 1 5}$ \\
24 Solicita su inclusión en el Aula de Convivencia & 13 & 10.48 & 2.61 \\
\hline
\end{tabular}

Las medidas también se agruparon en factores mediante análisis factorial. Aunque el término estadístico «factor» se utiliza tanto para CCC como medidas aplicadas es necesario señalar que son variables distintas. En la tabla 3 se presentan las medidas adoptadas por el profesorado, organizadas por factores junto a la frecuencia de uso de los mismos y su efectividad media. A su vez se incluyen las medidas incluidas en cada factor y la efectividad media de cada una. Los factores de medidas más frecuentemente utilizados son los de «gestión de aula» (94.35\%) y «traslado de autoridad» (85.48\%).

Los factores de medidas más eficaces son «acciones punitivas» con una valoración media de 3.07, y «estrategia organizativa» con 2.90 de valoración. El factor de medida «mediación» aparece como el menos utilizado y el menos eficaz.

Frecuenciay efectividad percibida de las medidas adoptadas por el profesor para cada factor CCC

En la tabla 4 pueden observarse las frecuencias y efectividad de los factores de medida para cada factor de conducta. Para las conductas de «desafío y acoso» se utiliza con mayor frecuencia las medidas de «traslado
Tabla 4

Frecuencia y efectividad percibida de los factores de medidas para cada factor de conducta. NOTA: Tanto las frecuencias de las medidas como su respectiva efectividad media están calculadas para los casos en los que un problema de conducta tiene una frecuencia alta, media o baja. Cuando una conducta tiene una frecuencia nula, obviamente no fue considerada para el presente análisis.

\begin{tabular}{|c|c|c|c|}
\hline \multirow{2}{*}{ Factores de conductas y medidas } & \multicolumn{2}{|c|}{ Frecuencia } & \multirow{2}{*}{$\begin{array}{l}\text { Efectividad } \\
\text { Media (1-4) }\end{array}$} \\
\hline & $\mathbf{n}$ & $\%$ & \\
\hline \multicolumn{4}{|l|}{ Desafío y acoso } \\
\hline Gestión de aula & 98 & $79.03 \%$ & 3.06 \\
\hline Traslado autoridad & 100 & $80.65 \%$ & 2.88 \\
\hline Estrategia organizativa & 59 & $47.58 \%$ & 3.00 \\
\hline Acciones punitivas & 45 & $36.29 \%$ & 3.20 \\
\hline Mediación & 57 & $45.97 \%$ & 2.71 \\
\hline Varias & 77 & $62.10 \%$ & 2.84 \\
\hline \multicolumn{4}{|l|}{ Déficit de atención } \\
\hline Gestión de aula & 114 & $91.94 \%$ & 2.90 \\
\hline Traslado autoridad & 76 & $61.29 \%$ & 2.73 \\
\hline Estrategia organizativa & 63 & $50.81 \%$ & 2.86 \\
\hline Acciones punitivas & 57 & $45.97 \%$ & 3.10 \\
\hline Mediación & 25 & $20.16 \%$ & 2.83 \\
\hline Varias & 84 & $67.74 \%$ & 2.80 \\
\hline \multicolumn{4}{|l|}{ Pasotismo } \\
\hline Gestión de aula & 95 & $76.61 \%$ & 2.78 \\
\hline Traslado autoridad & 53 & $42.74 \%$ & 2.69 \\
\hline Estrategia organizativa & 15 & $12.10 \%$ & 2.40 \\
\hline Acciones punitivas & 48 & $38.71 \%$ & 3.10 \\
\hline Mediación & 9 & $7.26 \%$ & 2.56 \\
\hline Varias & 46 & $37.10 \%$ & 2.80 \\
\hline \multicolumn{4}{|l|}{ Mal uso de materiales } \\
\hline Gestión de aula & 80 & $64.52 \%$ & 2.91 \\
\hline Traslado autoridad & 40 & $32.26 \%$ & 2.77 \\
\hline Estrategia organizativa & 16 & $12.90 \%$ & 2.94 \\
\hline Acciones punitivas & 20 & $16.13 \%$ & 3.48 \\
\hline Mediación & 16 & $12.90 \%$ & 2.75 \\
\hline Varias & 54 & $43.55 \%$ & 3.06 \\
\hline \multicolumn{4}{|l|}{ Agresión al profesor } \\
\hline Gestión de aula & 0 & $0.00 \%$ & \\
\hline Traslado autoridad & 2 & $1.61 \%$ & 3.50 \\
\hline Estrategia organizativa & 0 & $0.00 \%$ & \\
\hline Acciones punitivas & 0 & $0.00 \%$ & \\
\hline Mediación & 0 & $0.00 \%$ & \\
\hline Varias & 0 & $0.00 \%$ & \\
\hline
\end{tabular}

de autoridad» (80.65\%) y con mayor efectividad las «acciones punitivas» (3.20). Para las conductas de «déficit de atención», «pasotismo» y «mal uso de materiales» las medidas más utilizadas son las de «gestión de aula» (91.94\%, 76.61\% y 64.52\% respectivamente) y las más efectivas las «acciones punitivas» (3.10, 3.10 y 3.48 respectivamente).

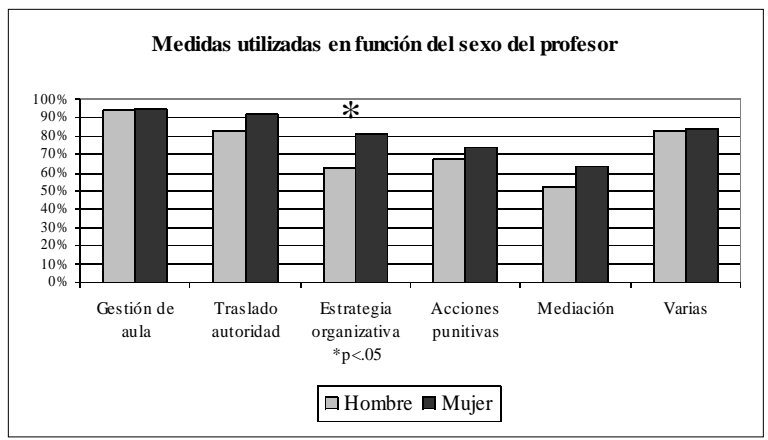

Gráfico 1

Medidas utilizadas en función del sexo del profesor.

Tabla 3

Denominación de los factores de medidas, junto con su frecuencia y efectividad media percibida. Medidas que los constituyen y su efectividad individual.

\begin{tabular}{|c|c|c|c|c|c|}
\hline Factores de Medida & \multicolumn{2}{|c|}{ Frecuencia } & $\begin{array}{l}\text { Efec. } \\
\text { Media }\end{array}$ & \multicolumn{2}{|c|}{$\begin{array}{l}\text { Medidas incluidas en cada factor y su efectividad } \\
\text { media }\end{array}$} \\
\hline \multirow{7}{*}{$\begin{array}{l}\text { Medidas de FACTOR 1: } \\
\text { GESTIÓN DE AULA }\end{array}$} & \multirow{7}{*}{117} & \multirow{7}{*}{$94.35 \%$} & \multirow{7}{*}{2.89} & 12 Diseña actividades & 2.78 \\
\hline & & & & 2 Llama al alumno por su nombre & 2.85 \\
\hline & & & & 9 Mira fijamente o hace algún gesto & 2.88 \\
\hline & & & & 1 Penaliza la nota & 2.78 \\
\hline & & & & 7 Reflexiona sobre la conducta & 2.68 \\
\hline & & & & 14 Refuerza positivamente & 3.05 \\
\hline & & & & 4 Se calla hasta que los alumnos & 3.13 \\
\hline \multirow{4}{*}{$\begin{array}{l}\text { Medidas de FACTOR 2: } \\
\text { TRASLADO AUTORIDAD }\end{array}$} & \multirow{4}{*}{106} & \multirow{4}{*}{$85.48 \%$} & \multirow{4}{*}{2.78} & 18 Deriva al Dpto. de Orientación & 2.60 \\
\hline & & & & 8 Envía a la Jefatura de Estudios & 3.12 \\
\hline & & & & 6 Lo explica al tutor para su trata & 2.55 \\
\hline & & & & 5 Redacta un parte & 2.86 \\
\hline \multirow{3}{*}{$\begin{array}{l}\text { Medidas de FACTOR 3: } \\
\text { ESTRATEGIA } \\
\text { ORGANIZATIVA }\end{array}$} & \multirow{3}{*}{85} & \multirow{3}{*}{$68.55 \%$} & \multirow{3}{*}{2.90} & 17 Cambia al/los alumnos de sitio & 2.85 \\
\hline & & & & 10 Expulsión temporal de clase & 3.07 \\
\hline & & & & 22 Ignora el hecho & 1.99 \\
\hline \multirow{3}{*}{$\begin{array}{l}\text { Medidas de FACTOR 4: } \\
\text { ACCIONES PUNITIVAS }\end{array}$} & \multirow{3}{*}{86} & \multirow{3}{*}{$69.35 \%$} & \multirow{3}{*}{3.07} & 11 Castiga con trabajo escrito & 2.79 \\
\hline & & & & 20 Castiga sin recreo o final jor & 3.35 \\
\hline & & & & 16 Llama personalmente al padre & 3.25 \\
\hline \multirow{4}{*}{$\begin{array}{l}\text { Medidas de FACTOR 5: } \\
\text { MEDIACIÓN }\end{array}$} & \multirow{3}{*}{68} & \multirow{3}{*}{$54.84 \%$} & \multirow{3}{*}{2.72} & 13 Actúa de mediador & 2.74 \\
\hline & & & & 21 Realiza contrato & 2.79 \\
\hline & & & & 24 Solicita su inclusi & 2.61 \\
\hline & \multirow{4}{*}{103} & \multirow{4}{*}{$83.06 \%$} & \multirow{4}{*}{2.89} & 23 Castiga con trabajo físico & 3.15 \\
\hline \multirow{3}{*}{$\begin{array}{l}\text { Medidas de FACTOR 6: } \\
\text { VARIAS }\end{array}$} & & & & 3 Establece charlas individuales & 2.83 \\
\hline & & & & 15 Grita por encima & 2.83 \\
\hline & & & & 19 Nombra responsabl & 3.19 \\
\hline
\end{tabular}

Relación entre las medidas adoptadas por el profesoradoycaracterísticas personales

En el análisis bivariante respecto a las medidas adoptadas por el profesor, podemos establecer que las profesoras usan con más frecuencia las medidas de «estrategia organizativa» (Gráfico 1), y que cuanto mayor es la antigüedad en el cuerpo menos frecuente es el uso de medidas «varias» (Gráfico 2). En ambos gráficos, «p» es el nivel de significación del test de chi-cuadrado. Al permitirse marcar varias medidas para una misma conducta los porcentajes suman más del 100\%.

\section{Discusión}

Los principales hallazgos del presente estudio muestran que las medidas adoptadas con mayor frecuencia han sido la de penalizar la nota (76.61\%), llamar al alumno por su nombre o preguntarle $(75,81 \%)$ y establecer charlas 




individuales y callarse hasta que los alumnos bajen el tono, ambas con una frecuencia del 74.19\%. A su vez, el factor de medidas más frecuentemente utilizado ha sido el de gestión de aula con un 94.35\%. Salvo para las CCC del factor denominado desafío y acoso, donde se utilizan mayoritariamente medidas de traslado de autoridad (80.65\%), para las CCC englobadas en los factores de déficit de atención, pasotismo y mal uso de materiales, las medidas más utilizadas son las de gestión de aula (91.94\%, 76.61\% y 64.52\% respectivamente). Características personales del profesor como el sexo y antigüedad en el cuerpo docente son variables que afectan al tipo de medidas utilizadas ante CCC del alumnado.

Frecuenciay efectividad percibida de las medidas adoptadas por el profesorado

Los resultados del presente estudio muestran una frecuencia de uso de los factores de medidas, ordenadas de la siguiente forma: en primer lugar gestión de aula, seguido de traslado de autoridad, varias, acciones punitivas, estrategia organizativa y por último las de mediación. Lo que parece indicar que el profesorado intenta solucionar la problemática en el aula como primera opción y, en caso de no resultar satisfactoria, decide acudir a otras medidas. También cabe destacar que el factor de medida más eficaz es el de acciones punitivas, seguido del de estrategias organizativas, gestión de aula, traslado de autoridad, varias y por último el de mediación. El factor de medida denominado «varias» alude a las medidas de castigar con trabajo físico, establecer charlas individuales, gritar por encima del alumnado y nombrar responsables para cada sesión.

La media denominada «castiga con trabajo físico», englobada dentro del factor de medidas denominado «varias», sólo ha sido utilizada en 15 ocasiones (un 12,10\%). Podemos entender que el profesorado evita generar una asociación entre ejercicio físico y castigo, evitando un condicionamiento aversivo al mismo. Consideramos que la utilización de esta medida debería ser nula o reducirse a situaciones excepcionales y siempre lejos de asociar ejercicio o actividad física con sufrimiento.

Ello nos muestra cómo el factor de medida percibido como más eficaz, acciones punitivas, no es el más utilizado, circunstancia que podríamos justificar en el intento prioritario del profesorado de solucionar las situaciones con medidas más integradoras y no exclusivamente sancionadoras. Sin embargo, una vez llegado el momento de su uso se constituyen como las más eficaces. Sería conveniente tener en consideración que el profesorado, en algunos casos, ha podido entender la «efectividad» de una medida como una acción de «efecto inmediato», lo que daría unos bajos resultados a las medidas contempladas bajo el factor de «mediación» y alto a las «punitivas», aunque también podrían intervenir otros condicionantes, como la formación del profesorado, los medios y tiempos disponibles para aplicar unas u otras medidas. Algo que justificaría afirmaciones como la de Binaburo \& Muñoz (2007) para quienes se hace necesaria la formación del profesorado en resolución de conflictos.

Los resultados de esta investigación y coincidiendo con Figueras et al. (2014) nos orientan a la necesidad de abordar el conflicto desde la perspectiva del conjunto de miembros adultos de la comunidad educativa y no reducirlo únicamente al colectivo de los profesores pues la participación de las familias y otros miembros de la comunidad en los centros escolares nos permitirán una perspectiva más global y una responsabilidad compartida. Paralelamente conviene utilizar grupos interactivos basados en la cooperación y heterogeneidad de las personas (alumnos, familias, otros miembros de la comunidad educativa y profesores) que pueden incidir de forma positiva en la disminución de los conflictos como nos proponen Buscà, Ruiz \& Rekalde (2014), Capllonch, Figueras \& Lleixà (2014) y Martín \& Ríos (2014).

Parece necesario continuar investigando en este aspecto, así como intentar profundizar en el análisis y relación entre las distintas CCC y las medidas utilizadas.

Es necesario considerar que la actuación del profesor debe procurar además de atajar la CCC del momento, mejorar la relación interpersonal con el alumnado e ir desarrollando en el mismo las habilidades adecuadas para poder dar una respuesta correcta a los distintos conflictos, a la vez que facilitar su integración en el grupo y el proceso de enseñanzaaprendizaje. Esta argumentación en las medidas a utilizar antela aparición de CCC en el aula, se relaciona directamente con los posibles factores preventivos vinculados a la persona (alumno) tales como: buscar la mejora de la autoestima, desarrollar el pensamiento reflexivo, crítico, escucha activa y las habilidades de asertividad, adquisición y mejora de habilidades sociales y de comunicación, la empatía y la expresión emocional, fortalecer valores positivos como el respeto y la tolerancia entre los jóvenes, y conocimientos sobre la conducta violenta y resolución de conflictos (Ortega, Mínguez \& Saura, 2003; Estévez, 2005; Ortega \& Mora-Merchán, 2005; Ortega \& Del Rey, 2006; Binaburo \& Muñoz, 2007; Buccoliero, 2007; Cerezo, 2007b; Defensor del Pueblo, 2007; Fernández, 2007; Ruiz, Cerezo \& Esteban, 2007; Ruiz, 2008). Es importante destacar la importancia de desarrollar programas deeducación emocional y de expresión corporal, como señalan Padilla \& Coterón (2013) tras su estudio en relación a la intervención con programas de danza.

Así mismo, debemos considerar los factores preventivos vinculados a las relaciones destacando el tratamiento respetuoso e individual del alumnado, la atención a la diversidad, mantener actitudes cálidas y de ayuda, introducir elementos de reflexión social sobre los conflictos, conseguir un clima de aula positivo, seguro, ordenado, comunicativo, generador de expectativas de éxito. Planificar trabajo en grupo cooperativos, fomentar la mediación y establecer unas normas de aula

\begin{tabular}{|c|c|c|}
\hline \multicolumn{3}{|c|}{ Estrategias para actuar ante la disrupción y/o los problemas de comportamiento en clase } \\
\hline Lera et al. (2007) & Moreno \& Torrego (2007) & Rincón (2002) \\
\hline $\begin{array}{l}\text { 1. Ser oportuno. Abordar el comportamiento problema } \\
\text { tan pronto sea posible. } \\
\text { 2. Desarrollo de un plan de reglas y derechos. } \\
\text { Desarrollar un plan acordado con el alumnado sobre } \\
\text { cinco reglas (no más) que hay que intentar cumplir. Se } \\
\text { premia cuando se hayan conseguido (bien } \\
\text { individualmente o grupalmente). } \\
\text { 3. Adecuación. Asegurarse de que las propias reacciones } \\
\text { parezcan razonables frente al comportamiento } \\
\text { problemático. Es importante no escalar en el conflicto y } \\
\text { si este fuese intenso al principio, bajar el nivel, sonreír, } \\
\text { agradecer y mirar a los ojos. } \\
\text { 4. Reactividad. Hablar con el alumno después de la } \\
\text { clase y llegar a acuerdos de qué hacer en la siguiente } \\
\text { sesión, conversar sobre los posibles comportamientos } \\
\text { alternativos. }\end{array}$ & $\begin{array}{l}\text { 1. Atender específicamente según la persona } \\
\text { disruptiva, hay alumnado al que se debe } \\
\text { responder y otro al que hay que ignorar. } \\
\text { 2. Anticiparse a las conductas, variación de } \\
\text { estímulos para evitar que llegue a haber } \\
\text { disrupción. } \\
\text { 3. Transferir responsabilidades. } \\
\text { 4. Hablar individualmente con el alumno. } \\
\text { 5. No transmitir agresividad. } \\
\text { 6. Que sea el grupo quien discuta y resuelva. } \\
\text { 7. Buscar el motivo de la conducta. } \\
\text { 8. Conseguir un momento de respiro tanto } \\
\text { para el alumno disruptivo como para el propio } \\
\text { profesor. } \\
\text { 9. Evitar considerarla una agresión personal. }\end{array}$ & $\begin{array}{l}\text { 1. Verlo todo, corregir poco y castigar muy poco. Se debe corregir o aconsejar, y } \\
\text { castigar únicamente en extrema necesidad y con garantía de éxito. A veces será } \\
\text { muy oportuno posponer razonamiento sobre lo ocurrido hasta que las personas } \\
\text { implicadas hayan recuperado su equilibrio anímico. } \\
\text { 3. “Programar" la disciplina. Si el educador reflexiona sobre las circunstancias } \\
\text { que concurren habitualmente en el grupo de alumnos, con cierta facilidad precisará } \\
\text { la conexión entre determinados hechos -desordenes, griterío, protestas-y las } \\
\text { circunstancias causales que los provocaron. En general, previenen la disciplina los } \\
\text { factores siguientes: puntualidad, agilidad en el inicio de las tareas, buena } \\
\text { preparación de las actividades y de los materiales necesarios, delegar tareas } \\
\text { rutinarias, distribución de cargos para el caso de incidentes (pintura en el suelo, } \\
\text { vidrio roto, accidente,...), evitar comparaciones, reflexionar sobre lo ocurrido en } \\
\text { ocasiones anteriores, seguimiento cercano de determinados alumnos, promover el } \\
\text { éxito de todos en alguna ocasión, comunicar las normas. }\end{array}$ \\
\hline
\end{tabular}


claras y compartidas por el grupo garantizando su mantenimiento y refuerzo en el tiempo, fomentar la toma de decisiones por parte del alumnado y, en alguna ocasión, dar la oportunidad a los intereses y gustos del alumnado en la gestión del aula o materia, así como considerar intervenciones en los diferentes niveles en que se desarrolla el estudiante (Gómez, 2002; Rincón, 2002; Orte, Ballester \& Oliver, 2003; Supaporn, Dodds \& Griffin, 2003; Estévez, 2005; Ortega \& Mora-Merchán, 2005; Consejo Escolar deAndalucía, 2006; Aznar et al., 2007; Binaburo \& Muñoz, 2007; Buccoliero, 2007; Cerezo, 2007b; Defensor del Pueblo, 2007; Fernández, 2007; Moreno, Moreno, Sicilia, Martínez, \&Alonso, 2008; Ruiz, 2008; Gómez \& Ordoñez, 2014; Valdés \& Martínez, 2014; Mejía \& Urrea, 2015).

Rincón (2002), Lera, Jensen \& Josang (2007) y Moreno \& Torrego (2007) aportan algunas estrategias para actuar ante la disrupción y/o los problemas de comportamiento en clase (Cuadro 1).

Frecuencia y efectividad percibida de las medidas adoptadas por el profesor para cada factor CCC

Los resultados del presente estudio muestran que para las conductas de «desafío y acoso» se utiliza con mayor frecuencia las medidas de «traslado de autoridad» (80.65\%) y con mayor efectividad las «acciones punitivas» (3.20). Para las conductas de «déficit de atención», «pasotismo» y «mal uso de materiales» las medidas más utilizadas son las de «gestión de aula» (91.94\%, 76.61\% y 64.52\% respectivamente) y las más efectivas las «acciones punitivas» (3.10, 3.10 y 3.48 respectivamente).

En este análisis bivariante y teniendo en cuenta la utilización de los factores demedida para cada factor de conducta en función dela frecuencia de estos últimos, se puede establecer que cuando se dan con frecuencia conductas de «desafío y acoso» se suelen usar más medidas «organizativas». Y cuando se dan con frecuencia conductas de «déficit de atención» se suelen utilizar más medidas «varias», ocurriendo lo mismo con las medidas «organizativas», aunque en este caso la asociación no llega a alcanzar la significación estadística.

Es necesario destacar que es imprescindible acudir a la relación entre factores de medidas y de conductas para poder interpretar con garantía los datos. Así, un factor de medida podría ser muy efectivo para un factor de conducta y no serlo para otro. Sin embargo, hay que seguir luchando con respuestas eficaces que permitan mantener y conservar la convivencia pacífica y el respeto a los demás, que son la clave de una sociedad democrática(Villagrasa, 2012).Asu vez, debemos considerar que algunas medidas, si conllevan corrección o sanción, son sanciones administrativas que aunque pretendiento un efecto educativo sobre el alumno, implican ciertas peculiaridades en la aplicación de algunos de los principios que rigen el derecho sancionador, tales como el principio de legalidad(García, 2014).

Relación entre las medidas adoptadas por el profesorado y características personales

Las profesoras usan con más frecuencia las medidas de «estrategia organizativa», mientras que cuanto mayor es la antigüedad en el cuerpo menos frecuente es el uso de medidas «varias». Szymanska \& Timmermans (2007), citando a Huberman (1993), consideran dentro de las secuencias profesionales que en la segunda fase, de estabilización, comprendida entre los cuatro y seis años de antigüedad, es cuando el docente ya tiene suficientes habilidades educativas para sentirse un líder y un efectivo docente. Por tanto, resultan fundamentales las competencias de trabajo en grupo y de manera colaborativa entre el profesorado (Pazo \& Tejada, 2012) al objeto de compartir responsabilidades, aumentar la reflexión y el análisis colectivo. Ello ayudaría a los profesores con menos antigüedad en el cuerpo a dominar estrategias de gestión que son eficaces para los compañeros más experimentados.

La investigación educativa demuestra que las variables de lo que llamamos gestión y organización del aula son las que más influyen y determinan no sólo la frecuencia de aparición de conductas antisociales, sino también el aprendizaje de los alumnos, su rendimiento académico y la «salud» de las relaciones interpersonales (Jares, 2006). Es por ello que la formación y actualización docente debe ser continua y eficaz, en base a que los conflictos son parte integrante de la profesión (Fernández, 2007) y el profesor debe disponer y conocer de estrategias y líneas de actuación sobre los factores que intervienen en el proceso de enseñanzaaprendizaje (Rosales, 2004; González, 2005).

De una forma general podemos afirmar que las respuestas del profesorado encuestado coinciden con lo que, en base a la revisión bibliográfica realizada, podríamos denominar como una buena gestión de aula. Entre ellas se destaca la conveniencia de permitir a los estudiantes influir sobre sus propios procesos de aprendizaje, saber siempre qué está pasando, reaccionar de forma apropiada ante situaciones críticas, ser capaz de cambiar y organizar cuando las cosas no van bien, usar claves no-verbales, alabar las conductas positivas, no dejar que un comportamiento leve interrumpa el trabajo, organizar las actividades en secuencias sucesivas, resolver los problemas en niveles bajos, plantear el trabajo en grupo y preguntar al alumnado por sus intereses. Los resultados nos orientan hacia la conveniencia del modelo dialógico y comunitario de prevención de conflictos propuesto por Aubert, Bizkarra \& Calvo (2014), Capllonch, Figueras \& Lleixà (2014) y Buscà, Ruiz \& Rekalde (2014) superando el modelo disciplinar basado en una autoridad y la aplicación de medidas sancionadoras y al modelo mediador dependiente de alguna persona experta.

\section{Conclusiones}

Tras del desarrollo del presente estudio proponemos unas pautas de intervención genéricas de medidas ante las CCC. En primer lugar, con el objetivo de tratar el conflicto en el aula a la vez quemejorar la integración del alumno y las relaciones interpersonales se aplicarían medidas de «gestión de aula» y «organizativas»; posteriormente, si fuese necesario, se reforzaría la intervención por medio de técnicas o documentos específicos de «mediación»; y por último, cuando las medidas anteriormente aplicadas no han resultado satisfactorias procederíamos a trasladar la intervención a un tercer agente y/o la aplicación de medidas punitivas que se consideren eficaces, por tanto, utilizaríamos medidas de los factores denominados de «traslado de autoridad» $\mathrm{y}$ «acciones punitivas».

En casos de CCC graves, como agresiones físicas, acoso o similar, siempre será necesario comunicarlo a jefatura de estudios y tutoría, por el procedimiento que el centro tenga establecido en su reglamento de organización y funcionamiento. Es necesario indicar que estas medidas y procedimientos están interrelacionados y, dependiendo del caso particular, de las características del alumno, de la situación específica, deben ser utilizados en función del criterio del profesor.

Aparecen enfrentados dos conceptos que viajan juntos en la convivencia de los centros educativos y en la gestión del aula, por un lado la necesidad de atajar con prontitud la CCC correspondiente para poder continuar desarrollando el proceso de enseñanza-aprendizaje de todo el grupo y, por otro, la conveniencia de educar a partir del conflicto y CCC aparecida en clase.

\section{Referencias}

Aubert,A., Bizkarra, M. \& Calvo, J. (2014). Actuaciones educativas de éxito desde laEducación Física. RETOS. Nuevas tendencias en Educación Física, Deporte y Recreación, 25, 144-148.

Aznar, I., Cáceres, Ma \& Hinojo, Fco.(2007). Estudio dela violencia y conflictividad escolar en las aulas de Educación Primaria a través de un cuestionario de clima de clase: El caso de las provincias de Córdoba y Granada (España). Revista Electrónica Iberoamericana sobre Calidad, Eficacia y Cambio en Educación, 5(1). Recuperado dehttp://www.rinace.net/reicepres.htm

Bancotovska, S.N.(2015). The attitudes and opinions of teachers to their competences. International Journal of Cognitive Research in Science, Engineering and Education:(IJCRSEE), 3(1), 99-103.

Binaburo, J. A. \& Muñoz, B. (2007). Educar desde el conflicto. Guía para la 
mediación escolar. Junta deAndalucía. Consejería de Educación.

Blaya, C., Debarbieux, E., Rey, R. \& Ortega, R. (2006). Clima y violencia escolar. Un estudio comparativo entre España y Francia. Revista de Educación, 339 293-315.

Buccoliero, E. (2007). Clima social. Recuperado de www.golden5.org/programa.

Buscà, F., Ruiz, L. \& Rekalde, I. (2014). Tratamiento del conflicto en las Comunidades deAprendizaje a través de la educación física. RETOS. Nuevas tendencias en Educación Física, Deportey Recreación, 25, 156-161.

Cassullo, G L. \& García, L. (2015). Estudio Delas Competencias Socio Emocionales y su Relación con el Afrontamiento en Futuros Profesores de Nivel Medio Revista Electrónica Inteuniversitaria de Formación del Profesorado, 18(1) 213-228. Recuperado de: DOI: http://dx.doi.org/10.6018/reifop.18.1.193041

Capllonch, M., Figueras, S. \& Lleixà, T. (2014). Prevención y resolución de conflictos en educación física: estado de la cuestión. RETOS. Nuevas tendencias en Educación Física, Deporte y Recreación, 25, 149-155.

Cerezo, F. (2007). La violencia en las aulas. Análisis y propuestas de intervención. Madrid: Pirámide.

Cerezo, F. (2007b). Conductas agresivas en la edad escolar. Aproximación teórica y metodológica. Propuestas de intervención. Pirámide. Madrid.

ComitéMixto OIT/UNESCOdeExpertos sobre laaplicacióndelas Recomendaciones relativas al personal docente-CEART-(1996). Recomendación relativa a la situación del personal docente. Recuperado de http://portal.unesco.org/es/

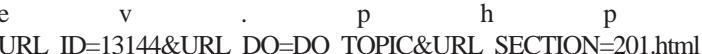

Consejo Escolar deAndalucía(2006). Encuesta sobre el estado de la Convivencia en los centros educativos. Recuperado de www.juntadeandalucia.es/educacion/ scripts/w cea/encuestas.htm

Defensor del Pueblo (2007). Violencia escolar: el maltrato entre iguales en la Educación Secundaria Obligatoria 1999-2006. Recuperado de http:// www.defensordelpueblo.es

Delceva, J. (2014). Classroom management. International Journal of Cognitive Research in Science, Engineering and Education, 2(1).

Delgado, M.A. (1991). Los Estilos de Enseñanza en la Educación Física. Propuesta para una Reforma de la Enseñanza. Granada: Universidad de Granada.

Estévez, E. (2005). Violencia, victimización y rechazo escolar en la adolescencia. Tesis doctoral. Universidad de Valencia.

Fernández, I. (2007). Prevención de la Violencia y Resolución de Conflictos. El clima escolar como factor de calidad. Edo Narcea. Madrid.

Fernández, J. M. (2015). Convivencia escolar. Un estudio en la provincia de Sevilla (España) International Journal of Educational Research and Innovation(IJERI), 3, 78-93.

Figueras, S., Calvo, J. \& Capllonch, M. (2014). Prevención y resolución del conflicto en educación física desde la perspectiva de los adultos miembros de la comunidad educativa en las comunidades de aprendizaje. Retos. Nuevas tendencias en Educación Física, Deportey Recreación, 25, 168-173.

García, M.J. (2014). La imposición de prestaciones personales en concepto de sanción en el ámbito educativo». Revista catalana de dret públic, 49, 130-150 DOI: $10.2436 / 20.8030 .01 .36$

Gavotto, O. (2015). Actuaciones disruptivas en educación secundaria: un análisis paramejorarla convivencia escolar. Revista Iberoamericana para la Investigación y el Desarrollo Educativo, 5(10). Recuperado de http://www.ride.org.mx/ index.php/RIDE/article/view/108

Gómez,P. (2002). Prevención de conductas violentas en niños/as y jóvenes. Revista Electrónica Interuniversitaria de Formación del Profesorado, 5(2). Recuperado dehttp://www.aufop.com/aufop/revistas/arta/digital/107/789

Gómez, J.A. \& Ordoñez, O. (2014). Prácticas disciplinarias escolares: aproximación conceptual para un proyecto con estudiantes, docentes y acudientes. Revista Académica e Institucional Páginas de la UCP, 95, 39-60.

González, P.J.(2005). Propuestas para afrontar algunas dificultades propias delárea de Educación Física. Quaderns Digitals, 40. Recuperado de http:www.quadernsdigitals.net

Jares, X. (2006). Conflicto y Convivencia en los centros educativos de secundaria. Revista de Educación, 339, 467-491.

Lera, M. J. Jensen, K. \& Josang, F. (2007). Gestión del aula. Recuperado de www.golden5.org/programa.

Luengo, F. \& Sánchez, P, (2006). RRI, elaboración de normas y consecuencias. Convivencia en la escuela. Herramientas de trabajo para el profesorado, 2, 1

Macías, D.F \& Ariel, J.(2015). Classroom Management:A Persistent Challenge for Pre-Service Foreign Language Teachers. PROFILE 17(2), 81-99. Recuperado dehttp://www.revistas.unal.edu.co/index.php/profile/article/view/43641

Martín, M. \& Ríos, O. (2014). Prevención y resolución del conflicto en educación física desdelaperspectiva del alumno. RETOS. Nuevastendencias en Educación Física, Deporte y Recreación, 25, 162-167.

McCormack, A. (1997). Classroom Management Problems, Strategies and Influences in Physical Education. European Physical Education Rewiew, 3(2), 102-115. Mejía, C. \& Urrea A. (2015). La confianza en el aula como eje transformador del mundo escolar. Sophia, 11(2), 223-236.

Moreno, J. M. \& Torrego, J.C. (2007). Mejora de los procesos de gestión del aula por parte del profesorado: interacción verbal y no verbal, discurso docente, estilo motivacional y respuesta inmediata a la disrupción en el aula (documento ProyectoAtlántida). Recuperadodehttp://www.cepcampgib.org/moodle1/mod/ resource/view.php?id=120\&subdir=/Organizacion_del_aula

Moreno, J.A., Cervelló, E., Martínez, C. \& Alonso, N. (2007). Los comportamientos de disciplina e indisciplina en Educación Física. Revista Iberoamericana de Educación, 44, 167-190.

Moreno, J.A., Sicilia,A, Martínez, C. \& Alonso, N.(2008). CoEducación y climas de aprendizaje en Educación Física. Aportaciones desde la teoría de Metas de Logro. Revista Internacional de Ciencias del Deporte, 11, 42-64.

Muñoz, J.M., Carreras, Mª \& Braza, P. (2004). Aproximación al estudio de las actitudes y estrategias de pensamiento social y su relación con los comportamientos disruptivos en el aula en la Educación secundaria. Anales de psicología, 20(1), 81-91.

Orte, C., Ballester, L. \& Oliver, J. L. (2003). Estrategias para la resolución de conflictos. Revista Electrónica Interuniversitaria de Formación del Profesorado, 6(2). Recuperado de http://www.aufop.com/aufop/revistas/lista/digital

Ortega, R. \& Del Rey, R(2006). La mediación escolar en el marco dela construcción de la Convivencia y la prevención de la violencia. Revista de la asociación de Inspectores de Educación de España, 2. Recuperadode http://sauce.pntic.mec.es/ ipul0002/AVANCES_REVISTA/adide02/2art_11.htm

Ortega, R. \& Mora-Merchán J.A. (2005). Conflictividad y violencia en la escuela. Diada. Sevilla.

Ortega, P., Mínguez, R. \& Saura, P. (2003). Conflictos en las aulas. Editorial Ariel. Barcelona.

Padilla, C. \& Coterón, J. (2013). ¿Podemos mejorar nuestra salud mental a través dela danza?: una revisión sistemática. Retos. Nuevas tendencias en Educación Física, Deporte y Recreación, 24, 167-170.

Pazo, C.I. \& Tejada, J. (2012). Las competencias profesionales en Educación Física. Retos. Nuevas tendencias en Educación Física, Deporte y Recreación, 22, 5-8.

Penalva, A., Hernández, M.A. \& Guerrero, C. (2013). La gestión eficaz del docente en el aula. Un estudio de caso. Revista Electrónica Interuniversitaria de Formación del Profesorado, 16(2), 77-91. Recuperado de http://dx.doi.org/ 10.6018/reifop.16.2.180931

Real Academia Española (22a edición) (2015). Recuperado de www.rae.es

Rajadell, N. (2002). La importancia de las estrategias docentes para la resolución de conflictos en el aula. Revista Electrónica Interuniversitaria de Formación del Profesorado,5(3). Recuperado de http://www.aufop.com/aufop/revistas/lista/ digital

Rincón, B. (2002). Variables que sustentan la Convivencia en los centros educativos

Revista electrónica Interuniversitaria de Formación del Profesorado,5(1). Recuperado de http://www.aufop.com/aufop/revistas/lista/digital

Ruiz, C., Cerezo, F. \& Esteban, M.(2007). El aula, marco institucional de socialización, interacción e integración. En Cerezo, F. (2007)Conductas agresivas en la edad escolar. Aproximación teórica y metodológica. Propuestas de intervención. Pirámide. Madrid.

Ruiz, J.C. (2008). Educación Física, valores éticos y resolución de conflictos: reflexiones y propuestas de acción. En Fraile, A.; López, V.M.; Ruiz, J. y Velázquez, C. (2008) La resolución de los conflictos en y a través de la Educación Física. GRAÓ. Barcelona.

Rosales,A. (2004). Estrategias didácticas o de intervención docente en elárea de la Educación Física. Revista Digital, 10(75). Recuperado de http:// www.efdeportes.com/efd75/estrateg.htm

Supaporn, S.; Doods, P. \& Griffin, L. (2003). An Ecological Analysis of Middle School Misbehavior Through Student and Teacher Perspectives. Journal of teaching in physical education, 22, 328-349.

Szymanska, J. \& Timmermans, J. (2007). Construyendo relaciones. Recuperado de www.golden5.org/programa.

Valdés,A., \& Martínez, E.A.(2014). Relación entre el autoconcepto social, el clima familiar y el clima escolar con el bullying en estudiantes de secundaria.Avances en Psicología Latinoamericana, 32(3), 447-457. Recuperado de http:// revistas.urosario.ed.com/index.php/apl/article/view/2697

Vallory, E. (2015). Escuelas por el aprendizaje la formación de los educadores y su nuevo rol. Revista Padres y Maestros / Journal of Parents and Teachers, 362, 64-71. Recuperado de: DOI: http://dx.doi.org/10.14422/pym.i362.y2015.011

Villagrasa, C. (2012). Los conûictos de derechos en el aula y las alternativas de gestión y resolución. Educatio Siglo XXI,30(2), 149-166. Recuperado dehttp:/ /revistas.um.es/educatio/article/view/153731/140771

Villalta, M. A. (2014). Organización escolar y trabajo de enseñanza en aula de establecimientos de alto desempeño educativo. Universitas Psychologica,13(1). Recuperado de doi:10.11144/Javeriana.UPSY13-1.oete 